\title{
Resuscitative Endovascular Balloon Occlusion of the Aorta: Focusing on the Procedure
}

\author{
Dong Hun Kim¹, Sung Wook Chang ${ }^{2}$ \\ 'Department of Surgery, Trauma Center, Dankook University Hospital, Cheonan, Korea \\ ${ }^{2}$ Department of Thoracic and Cardiovascular Surgery, Trauma Center, Dankook University Hospital, Cheonan, Korea
}

Resuscitative endovascular balloon occlusion of the aorta (REBOA) has been reported as an effective and minimally invasive damage control technique for life-threatening abdominal or pelvic exsanguination. Although high-quality evidence regarding REBOA is still needed, it is important to understand the procedure and be able to put it into practice clinically. Thus, here we demonstrate the procedure of REBOA through a video clip which is based on two cases of successful REBOA.

Key Words: Trauma; Resuscitation; Aorta; Balloon Occlusion; Hemorrhage

(Trauma Image Proced 2017(2):92-93)

\section{CASE}

Resuscitative endovascular balloon occlusion of the aorta (REBOA) was successfully performed in two patients with life-threatening abdominal and pelvic bleeding as a result of blunt trauma. Both patients were admitted with unstable hemodynamics and massive abdomino-pelvic bleeding. Systolic blood pressure just before REBOA was less than $80 \mathrm{mmHg}$. A 75 -year-old man had a ruptured spleen from a pedestrian traffic accident, and a 48-year-old man had an unstable pelvic fracture from an injury due to an 8-m fall. Both patients underwent REBOA with a targeted aortic location, accordant to zone I or III, in the emergency room (Video.) and then other damage control procedures or surgery was performed with permissive hypotension.

\section{DISCUSSION}

Two aortic zones are usually targeted for REBOA. Zone $\mathrm{I}$ is defined as a descending thoracic aorta from the origin of the left subclavian artery to the above part of the celiac artery for massive abdominal hemorrhage while zone III is as a infrarenal abdominal aorta above the aortic bifurcation for unstable pelvic hemorrhage (1). Using a 7-Fr balloon catheter (RESCUE BalloonTM, Tokai Medical Products, Aichi, Japan), access to the femoral artery can be started using the Seldinger technique or ultrasound guidance. A 7-Fr introducer sheath and 0.025-inch guide wire accordant to the balloon catheter are needed. After positioning the balloon in an appropriate aortic zone for temporary abdomino-pelvic bleeding, placement is confirmed with a fluoroscopy or serial radiography following balloon inflation with a mixture of saline and contrast. Partial

Received: September 7, 2017 Revised: October 13, 2017 Accepted: October 20, 2017

Correspondence to: Dong Hun Kim, Department of Surgery, Trauma Center, Dankook University Hospital, 201 Manghyang-ro, Dongnam-gu, Cheonan, Chungnam 31116, Korea

Tel: 82-41-550-7661, Fax: 82-41-550-0039, E-mail: saint7331@gmail.com

Copyright (C) 2017 Korean Association for Research, Procedures and Education on Trauma. All rights reserved.

@) This is an open-access article distributed under the terms of the Creative Commons Attribution Non-Commercial License (http://creativecommons.org/ licenses/by-nc/4.0) which permits unrestricted noncommercial use, distribution, and reproduction in any medium, provided the original work is properly cited 
aortic occlusion with permissive hypotension is then established with an intermittent aortic balloon deflation to decrease distal ischemia and perfusion injury when feasible $(2,3)$.

\section{Conflicts of Interest Statement}

None of authors have a conflict of interest.

\section{REFERENCES}

1. Stannard A, Eliason JL, Rasmussen TE. Resuscitative endovascular balloon occlusion of the aorta (REBOA) as an adjunct for hemorrhagic shock. J Trauma Acute Care Surg. 2011; 71:1869-72.
2. Johnson MA, Neff LP, Williams TK, DuBose JJ; EVAC Study Group. Partial resuscitative balloon occlusion of the aorta (P-REBOA): clinical technique and rationale. J Trauma Acute Care Surg. 2016;81(5 Suppl 2 Proceedings of the 2015 Military Health System Research Symposium):S133-7.

3. Napolitano LM. Resuscitative Endovascular Balloon Occlusion of the Aorta: Indications, Outcomes, and Training. Crit Care Clin. 2017 Jan;33(1):55-70.

\section{Video Legend}

Video. A video recording shows resuscitative endovascular balloon occlusion of the aorta using a 7-Fr balloon catheter in the emergency room. 